\title{
Hyperfragments from the lightest p-shell Hypernuclei
}

\author{
Olga Majlingová*† \\ Czech Technical University, Prague \\ E-mail: Dlga.majlingovadfs.cvut.cz

\section{Lubomir Majling} \\ Nuclear Physics Institute, Řežnear Prague and \\ Bogoliubov Laboratory of Theoretical Physics, JINR, Dubna, Russia \\ E-mail: majlingdujf.cas.cz
}

In this contribution, we discuss the potential of $\Lambda$-hypernuclear physics. A short overview of current experiments is given and the essential features of $\Lambda$ hypernuclei are briefly mentioned. The observation of the heavy hyper Hydrogen ${ }_{\Lambda}^{6} \mathrm{H}$ revived interest in identification of hyperfragments - products of the strong decay of a primary hypernuclei. The registration of hypernuclei ${ }_{\Lambda}^{6} \mathrm{H}$ and ${ }_{\Lambda}^{8} \mathrm{H}$ was already put on the list of tasks for Nuclotron. Theoretical background is pointed out and experimental possibilities are compared with other experiment. Our contribution is inspired by the possibility to identify hyperfragments in the reaction $\left(e, e^{\prime} K^{+}\right)$. In the near future two experiments: at JLab and at Mainz will provide a systematic study of the fragments from the primary $p$-shell hypernuclei. It is an important extension of the very successful experiments investigated discrete part of hypernuclear spectra. We present an approach to identification hyperfragments from $\mathrm{p}$-shell hypernuclei. As a first step we analyze the decay of the components of Strange Analogue States $\left(p_{\Lambda} p^{-1}, s_{\Lambda} s^{-1}\right)$ for hypernuclei $A \leq 9$ using Translation Invariant Shell Model (TISM). The attention is focused on the nuclear structure role of concrete decay channels population. Some predictions are given.

XXI International Baldin Seminar on High Energy Physics Problems,

September 10-15, 2012

JINR, Dubna, Russia

\footnotetext{
*Speaker.

$\dagger$ Work of L.M. was supported by grant P203/12/2126 of the Grant Agency of the Czech Republic. It is also part of the European Union Initiative FP7, Study of Strongly Interacting Matter (HadronPhysics3, SPHERE network). Work of O.M. was supported by Project LA08002 of the Ministry of Education, Youth and Sport of the Czech Republic.
} 


\section{Motivation}

Our work is inspired by the possibility to identify hyperfragments in the reaction $\left(e, e^{\prime} K^{+}\right)$. In the near future two experiments at JLab [W] and at Mainz [0] will provide a systematic study of the fragments from the primary $p$-shell hypernuclei. It is an important extension of the very successful experiments investigated discrete part of hypernuclear spectra [3]

The Proposal $[\mathrm{W}]$ intends to use high precision monochromatic $\pi^{-}$, from the unique two-body mesonic weak decay of hypernuclei to investigate light $\Lambda$-hypernuclei with variety of $(\mathrm{Z}, \mathrm{A})$ combinations through identification of hyperfragments from strongly produced hypernuclear continuum in $\left(e, e^{\prime} K^{+}\right)$electro-production. A collection of hypernuclei: neutron-rich hypernuclei, neutron drip line hypernuclei (including ${ }_{\Lambda}^{6} \mathrm{H}$ ) and mirror pairs of hypernuclei can be observed. The experiment will take a phase approach for the three different targets: ${ }^{7} \mathrm{Li},{ }^{9} \mathrm{Be}$, and ${ }^{12} \mathrm{C}$.

\section{Introduction}

In this contribution, we discuss the potential of $\Lambda$-hypernuclear physics. The subject involves many fields of modern theoretical and experimental physics, from nuclear structure to the fundamental constituents of matter and their interactions. The peculiar behavior of matter containing strange quarks has raised in recent years many interesting problems, one of which being the physics of hypernuclei. Hypernuclear physics was born in 1952, when the first hypernucleus was observed through its decays [ 困. Since then, it has been characterized by more and more new challenging questions and answers. The interest was further raised by the great advances made in the last years. Moreover, the existence of hypernuclei gives a new dimension to the traditional world of nuclei (states with new symmetries, selection rules, etc). They represent the first kind of flavored nuclei, in the direction of other exotic systems (e.g. charmed nuclei).

With the advent of the relativistic ion beams, they were used also for production of hypernuclei [ [6, 目], see below. Heavy ion collisions experiments at moderated energies will be performed at FAIR by the Compressed Baryonic Matter (CBM) Collaboration to study the nature of the transition between hadronic and quark matter. The very interesting result was announced recently by the STAR Collaboration at RHIC [ []]: the production of ${ }_{\Lambda}^{3} \mathrm{H}$ and antimatter hypernucleus $\overline{{ }_{\Lambda}^{3} \mathrm{H}}$ $(=\bar{n}+\bar{p}+\bar{\Lambda})$ was observed in $\mathrm{Au}+\mathrm{Au}$ collisions.

Hypernuclei not only bring a strangeness to nuclear physics, they provide a convenient laboratory for obtaining information about the hyperon-nucleon $(\mathrm{YN})$ interaction and explore the full SU(3) symmetry breaking baryon-baryon interaction strong and weak. In fact, the existing data on $\Lambda \mathrm{N}$ and $\Sigma \mathrm{N}$ strong interaction are extremely sparse and imprecise: production and scattering in the same target are required due to low hyperon beam intensities and short lifetimes. There are several realistic models for the free YN interactions, based on boson exchanges. Well-known are YN potentials of the Jülich group [ [ $]$ ] constructed along the same guidelines as used in the Bonn NN potential. The Nijmegen group [Q] for many years has developed several one-boson exchange potential models. They use SU(3) constrains on the coupling constants to fit about $4300 \mathrm{pp}+\mathrm{np}$ data on cross sections and variety of spin correlation together with 35 scattering $\Lambda \mathrm{N}$ and $\Sigma \mathrm{N}$ data at low energies. Since the empirical information on YN scattering consists almost exclusively on spin-averaged quantities like total and differential cross sections, the spin structure of the free $\mathrm{YN}$ 
interaction is essentially unknown. Therefore, various models for the $\mathrm{YN}$ interaction, which differ widely in their spin (and isospin) dependence, are able to describe the same scattering data. The only way to obtain low-momentum data is from hypernuclear spectroscopy: the results of hypernuclear structure calculations are sensitive to the spin dependence of the YN interaction and the finite-nuclei YN G-matrix provide a useful tool for testing the spin structure of the various YN

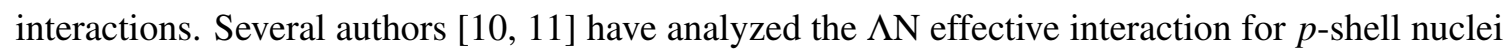
in terms of five phenomenological parameters $\mathrm{V}, \Delta, \mathrm{S}_{\Lambda}, \mathrm{S}_{\mathrm{N}}$, and $\mathrm{T}$. High precision data on light hypernuclei are of vital importance for the accurate determination of the missing parts of the YN interaction. Currently, there exists not only reliable methods for identification phenomenological strong interaction $\Lambda \mathrm{N}$, but also first attempts to relate it to modern Lattice QCD techniques [12]].

\section{Research in Europe}

Few years ago an European collaboration platform SPHERE (Strange Particles in Hadronic Environment Research in Europe) was established, to coordinate studies of hypernuclei at FINUDA (Frascati), KAOS (Mainz), HypHI (Darmstadt) and PANDA@FAIR. Now, it includes JPARC (Tokai) and CEBAF (JLab) also. FINUDA is still processing data from series of experiments exploring the production single hypernuclei with tagged stopped kaons [ㅈ]. The KAOS/A1 Collaboration at MAMI-C (Mainz) will produce hypernuclei by electron scattering [ [వ]. The HypHI experiment will investigate exotic hypernuclei which are produced by nuclear collision with stable heavy ion beams and rare isotope beams at GSI and FAIR [14]]. Similar experiments are envisaged at Nuclotron [15]]. The FLAIR - Collaboration at FAIR plans to produce strange nuclear systems by stopped antiprotons. Thanks to the use of $\bar{p}$ beams and the skillful combinations of experimental techniques, copious production of double $\Lambda$ hypernuclei is expected at the PANDA experiment which will enable high precision $\gamma$ spectroscopy for the first time [ए6].

The hypernuclear physics made an impressive progress in the last 40 years. First of all, the quest of hypernuclei production was solved partially: in one-step direct reactions such as

${ }^{A} \mathrm{Z} \quad\left(K^{-}, \pi^{-}\right) \quad{ }_{\Lambda}^{A} \mathrm{Z} \quad$ [ए]] and ${ }^{A} \mathrm{Z}\left(\pi^{+}, K^{+}\right){ }_{\Lambda}^{A} \mathrm{Z}$ [[18] $\Lambda$-hyperon replaces one neutron. Recently, reaction ${ }^{A} Z\left(e, e^{\prime} K^{+}\right){ }_{\Lambda}^{A}(Z-1)$, when proton is replaced by $\Lambda$ has been explored at JLab for the production of hypernuclei [प]]. The excellent resolution of $0.67 \mathrm{MeV}$ was attained.

The systematic study of excitation energy spectra were conducted for all $p$-shell hypernuclei, for some medium-heavy e.g. ${ }_{\Lambda}^{51} \mathrm{~V},{ }_{\Lambda}^{89} \mathrm{Y}$ as well as for ${ }_{\Lambda}^{208} \mathrm{~Pb}$. The $\Lambda$ single particle energies form a textbook example of single-particle behavior [20].

Recently, Tamura [2] solved the huge technical problems and constructed "Hyperball", a large acceptance Ge detector array dedicated to hypernuclear gamma-ray spectroscopy. A series of experiments on $p$-shell targets has been carried out at KEK and BNL using $\left(\pi^{+}, K^{+} \gamma\right)$ and $\left(K^{-}, \pi^{-} \gamma\right)$ reactions, respectively [[20].

Since the experiments performed to date used targets of stable nuclei, our knowledge is still limited to a small number of hypernuclei on/near the $\beta$-stability line.

On the chart of light p-shell nuclei and hypernuclei, Fig. W, we can see, that there exists much more hypernuclei than ordinary nuclei. $\Lambda$ hyperon stabilizes nuclear cores, acting as a glue. There are many particle-stable hypernuclei with unstable cores: ${ }_{\Lambda}^{6} \mathrm{He},{ }_{\Lambda}^{7} \mathrm{Be},{ }_{\Lambda}^{8} \mathrm{He},{ }_{\Lambda}^{9} \mathrm{Be}$. Many other hypernuclei with neutron excess may exist: e.g. ${ }_{\Lambda}^{6} \mathrm{H},{ }_{\Lambda}^{8} \mathrm{H},{ }_{\Lambda}^{11} \mathrm{Li}$. 


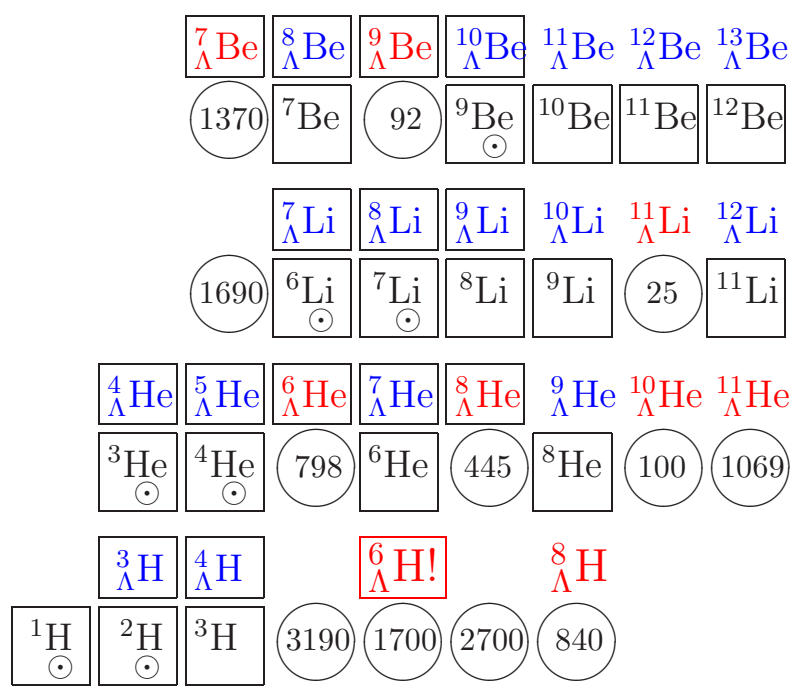

Figure 1: Chart of light p-shell nuclei and hypernuclei. In squares are already recognized hypernuclei; in the circles are resonances (unstable nuclei) with their instability in keV. Stable nuclei are marked by $\odot$.

In the last thirty years a new branch of nuclear physics, namely physics of nuclei in the vicinity of the neutron drip line has been constituted [D2]. Hypernuclear Physics could be used in the studies of the loosely bound nuclear systems, such are nuclei with neutron halo.

The hypernuclear facility FINUDA at the $\phi$-factory DA $\Phi$ NE (Frascati) [23] opened unprecedented possibilities and initiated great expectations. Very low energy of kaons from the $\phi$ decay allow the use of very thin solid stopping targets. The detector is designed to register both the $\pi^{-}$ from the formation reaction and charge products from the hypernuclear decay. We suggested [24] to study there strangeness and double exchange (S\&DCX) reaction $\left(K^{-}, \pi^{+}\right)$, which opens way to the production of neutron-rich hypernuclei. There are two paths how to arrive at the $\Lambda n p^{-2}$ states:

$$
\begin{array}{rlcl}
\text { either } & \text { by the pion charge exchange: } & K^{-} p \rightarrow \Lambda \pi^{0}, & \pi^{0} p \rightarrow n \pi^{+} \\
\text {or } & \text { through the } \Sigma N \rightarrow \Lambda N \text { conversion: } & K^{-} p \rightarrow \pi^{+} \Sigma^{-}, & \Sigma^{-} p \rightarrow \Lambda n .
\end{array}
$$

Unfortunately, both protons have to be in the same target nucleus, so cross sections are inevitably low. Nevertheless, the search for neutron-rich $\Lambda$ hypernuclei was one of the items on the FINUDA's list [25] and recently they succeeded.

The lightest unstable-core hypernucleus ${ }_{\Lambda}^{6} \mathrm{H}$ could be used as example, to demonstrate several different approaches and techniques that are actually used. It was predicted by Dalitz and Levi Setti [26], and has attracted attention even at "emulsion era" of hypernuclear physics. It could be formed in ${ }^{6} \mathrm{Li}-{ }^{7} \mathrm{Li}$-loaded emulsion: $K^{-}+{ }^{6} \mathrm{Li}\left({ }^{7} \mathrm{Li}\right) \rightarrow \pi^{+}+{ }_{\Lambda}^{6} \mathrm{H}+p(d)$. The three body decay mode ${ }_{\Lambda}^{6} \mathrm{H} \rightarrow \pi^{-}+t+t$ was searched for in [R7] but no decay at all was found.

The first attempt to observe ${ }_{\Lambda}^{6} \mathrm{H}$ (and other neutron-rich hypernuclei) was done at the DAФNE [28], but only upper limit was evaluated. Very recently, FINUDA Collaboration consider both production and decay in coincidence

$$
\begin{aligned}
K_{\text {stop }}^{-}+{ }^{6} \mathrm{Li} & \rightarrow{ }_{\Lambda}^{6} \mathrm{H}+\pi^{+}\left(\sim 250<p_{\pi^{+}}<255 \mathrm{MeV} / \mathrm{c}\right) \\
& \rightarrow{ }^{6} \mathrm{He}+\pi^{-}\left(\sim 130<p_{\pi^{-}}<137 \mathrm{MeV} / \mathrm{c}\right)
\end{aligned}
$$


and found three events corresponding unambiguously to the formation and subsequent decay of ${ }_{\Lambda}^{6} \mathrm{H}$ [Rg] with the result in $\mathrm{B}_{\Lambda}\left({ }_{\Lambda}^{6} \mathrm{H}\right)=4.0 \pm 1.1 \mathrm{MeV}$. This value compares well with our theoretical estimation [24].

The confirmation of the very existence of neutron-rich Hydrogen isotopes may be gain in experiments with relativistic hypernuclei here, in JINR. The first experiments with relativistic hypernuclei were done at Dubna many years ago [焑].

The advantages of the Dubna method are as follows [30]. Since hypernuclei (hyperfragments) in the experiments are produced by the excitation of high energy nuclei (up to $6 \mathrm{~A} \cdot \mathrm{GeV}$ ) the energy of the produced hypernuclei is just a little bit lower than that of an incident beam. In such a case, hypernuclei decay at tens $\mathrm{cm}$ beyond the target. For example, the mean hypernuclei decay range should be about $40-45 \mathrm{~cm}$. For experiments at the SPHERE spectrometer, it was suggested to observe hypernuclear decays in a vacuumed volume of $60 \mathrm{~cm}$ (approximately $70 \%$ of hypernuclei will decay inside of this volume). If the decay vertex was fixed inside of $60 \mathrm{~cm}$ path there is no doubt that the event was the decay of a hypernucleus since no background process can produce the vertex in vacuum, so one can obtain a pure sample of hypernuclei decays. Hypernuclei can be identified unambiguously. To be sure that the decay vertex was inside vacuum one must use an adequate tracker to measure the direction of secondary particles (to calculate the vertex coordinates). On the other hand, if an incident nuclear beam is not ${ }^{3} \mathrm{He}$, different hypernuclei and isotopes can be produced. While the charge of hypernuclei is measured with the trigger counters, isotopes can be identified by the daughter nuclei momentum measurement. Since the momenta of positive decay products should also be measured, a tracker should be installed beyond the analyzing magnet. Estimates show that the expected accuracy of the measurement of the vertex position and the daughter nuclei momenta is good enough to obtain sample of the safely identified hypernuclei.

It is suggested to start the hypernuclear research program with the investigation of ${ }_{\Lambda}^{4} \mathrm{H}$ and ${ }_{\Lambda}^{3} \mathrm{H}$ production in the helium beam. Such an experiment is similar to the previous one and allows one to check all systems of the set up including data processing. At the next step helium beam should be changed to the ${ }^{7} \mathrm{Li}$ beam to search for ${ }_{\Lambda}^{6} \mathrm{H}$ together with ${ }_{\Lambda}^{4} \mathrm{H}$ and ${ }_{\Lambda}^{3} \mathrm{H}$. The number of hypernuclear decays $(\mathrm{N})$ expected to be observed in 24 hours is given in Table $\mathrm{W}$. To discriminate the masses of the isotopes of the hypernuclear daughter nuclei one should measure the corresponding momenta. The momentum values of ${ }^{3} \mathrm{He},{ }^{4} \mathrm{He}$ and ${ }^{6} \mathrm{He}$ are concentrated in the $\approx 14 \mathrm{GeV} / \mathrm{c}, \approx 19 \mathrm{GeV} / \mathrm{c}$ and

Table 1: Production and decay

\begin{tabular}{|c|c|c|c|c|c|c|c|}
\hline beam & & target & & production & & decay & $\mathrm{N}$ \\
\hline${ }^{3} \mathrm{He}$ & + & ${ }^{12} \mathrm{C}$ & $\rightarrow$ & ${ }_{\Lambda}^{3} \mathrm{H}+\cdots$ & $\rightarrow$ & ${ }^{3} \mathrm{He}+\pi^{-}$ & 100 \\
\hline${ }^{4} \mathrm{He}$ & + & ${ }^{12} \mathrm{C}$ & $\begin{array}{l}\rightarrow \\
\rightarrow\end{array}$ & $\begin{array}{l}{ }_{\Lambda}^{4} \mathrm{H}+\cdots \\
{ }_{\Lambda}^{3} \mathrm{H}+\cdots\end{array}$ & $\begin{array}{l}\rightarrow \\
\rightarrow\end{array}$ & $\begin{array}{l}{ }^{4} \mathrm{He}+\pi^{-} \\
{ }^{3} \mathrm{He}+\pi^{-}\end{array}$ & 600 \\
\hline${ }^{7} \mathrm{Li}$ & + & ${ }^{12} \mathrm{C}$ & $\begin{array}{l}\rightarrow \\
\rightarrow \\
\rightarrow\end{array}$ & $\begin{array}{l}{ }_{\Lambda}^{6} \mathrm{H}+\cdots \\
{ }_{\Lambda}^{4} \mathrm{H}+\cdots \\
{ }_{\Lambda}^{3} \mathrm{H}+\cdots\end{array}$ & $\begin{array}{l}\rightarrow \\
\rightarrow \\
\rightarrow\end{array}$ & $\begin{array}{l}{ }^{6} \mathrm{He}+\pi^{-} \\
{ }^{4} \mathrm{He}+\pi^{-} \\
{ }^{3} \mathrm{He}+\pi^{-}\end{array}$ & 400 \\
\hline
\end{tabular}


$\approx 29 \mathrm{GeV} / \mathrm{c}$ bands correspondingly, see Fig $\square$. Such difference can be measured easily to separate three possible reactions of the hydrogen hypernuclei production and decay in the ${ }^{7} \mathrm{Li}$ beam.

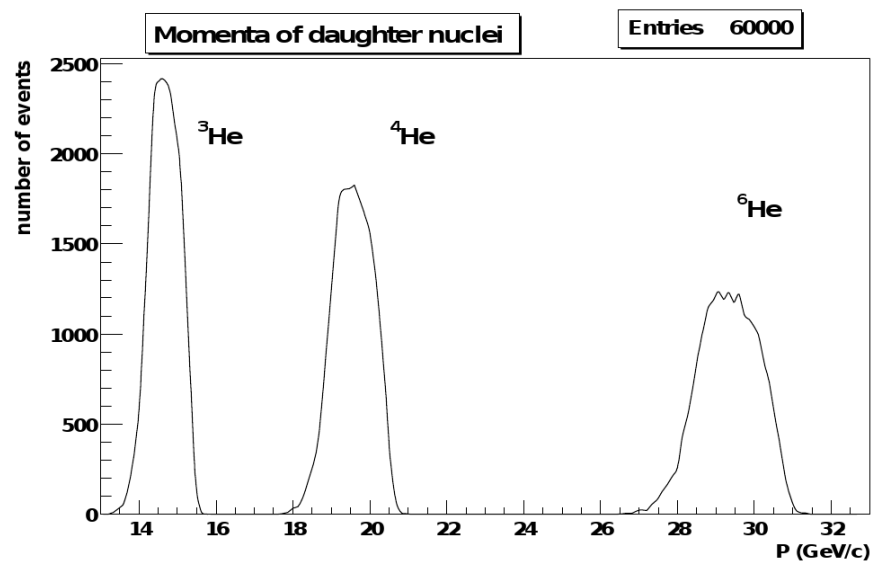

Figure 2: Momenta distributions of recoil nuclei ${ }^{3} \mathrm{He},{ }^{4} \mathrm{He}$ and ${ }^{6} \mathrm{He}$

We repeat once again advantages of Dubna setup presented at Baldin seminar six years ago [B]]: - unique identification of ${ }_{\Lambda}^{6} \mathrm{H}$ see Fig. ㅁ];

- possibility to measure lifetime of ${ }_{\Lambda}^{6} \mathrm{H}$ and to verify the existence of long-lived isomeric state in neutron-rich hypernuclei [B2];

- possibility to identify even extremely exotic hypernucleus ${ }_{\Lambda}^{8} \mathrm{H}(=p+\Lambda+\mathbf{6} n)$

- the chart of hypernuclei (Fig. W) suggests that $\Lambda$ might bind ${ }^{7} \mathrm{H}$ [B3] .

The hypernucleus ${ }_{\Lambda}^{6} \mathrm{H}$ may appear also as hyperfragment: proton decay of primary hypernucleus ${ }_{\Lambda}^{7} \mathrm{He}$ produced in reaction ${ }^{7} \operatorname{Li}\left(e, e^{\prime}, K^{+}\right)$. The Fig. B shows clearly why the highly excited states of ${ }_{\Lambda}^{7} \mathrm{He}$, in which the "inner proton" is substituted by $\Lambda$ (transition $p_{s} \rightarrow \Lambda_{s}$ ) are the source of hyperfragments ${ }_{\Lambda}^{4} \mathrm{H}$ and ${ }_{\Lambda}^{6} \mathrm{H}$. The thresholds for these decay channels are rather high, but large changes in the structure of these states prevent the neutrons or $\Lambda$ from emission.

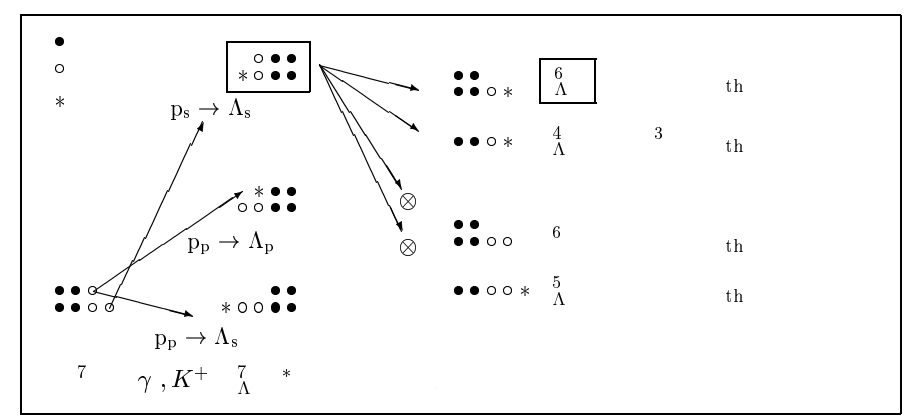

Figure 3: Different decay channels of ${ }_{\Lambda}^{7}$ He hypernucleus

So, it is the best candidate as "first-day target" for the Proposal [U] which intends to use high precision monochromatic $\pi^{-}$'s from the unique two-body mesonic weak decay of hypernuclei to investigate systematically light $\Lambda$-hypernuclei through identification of hyperfragments from strongly produced hypernuclear continuum (quasi free production) in $\left(e, e^{\prime} K^{+}\right)$reaction.

It is a great challenge to work out the model for description fragment's emission. 


\section{Approach}

In what follows, we proceed to build structure on top of foundations previously constructed.

Dalitz and Gal, in their seminal paper [34] consider the formation of $p$-shell hypernuclear states via $\left(K^{-}, \pi^{-}\right)$reactions and the prospects for $\gamma$-spectroscopy based on the decays of states $\mid s^{4} p^{k}: \alpha_{c} J_{c} T_{c}, s_{\Lambda}: \mathscr{J}>$. A comprehensive program for the shell model analysis of $\gamma$-ray transition in $p$-shell hypernuclei is conducted by Millener [35].

A natural extension of the shell model analysis are $\mid s^{4} p^{k}: \alpha_{c} J_{c} T_{c}, \underline{p_{\Lambda}}: \mathscr{J}>$ configurations. Simple calculations have been done [B] but it is important to include also $1 \hbar \omega$ states of core nucleus coupled to $s_{\Lambda}$. These configurations are necessary to permit the exclusion of spurious center-of-mass states from the shell model basis [37]].

The configurations $s^{-1} s_{\Lambda}$ e.g. $1 \hbar \omega$ excitations of core nucleus coupled to an $s_{\Lambda}$, were seen in spectra of lightest $p$-shell hypernuclei produced by strangeness exchange reaction $\left(K_{\text {in }}^{-}{ }_{\text {flight }}, \pi^{-}\right)$[38] , where we can see well defined unbound states with sufficiently narrow decay width. A nice example is hypernucleus ${ }_{\Lambda}^{6} \mathrm{Li}$, Fig. 田. In Ref. [39] we have discussed hypernucleus ${ }_{\Lambda}^{6} \mathrm{Li}$ in terms of an

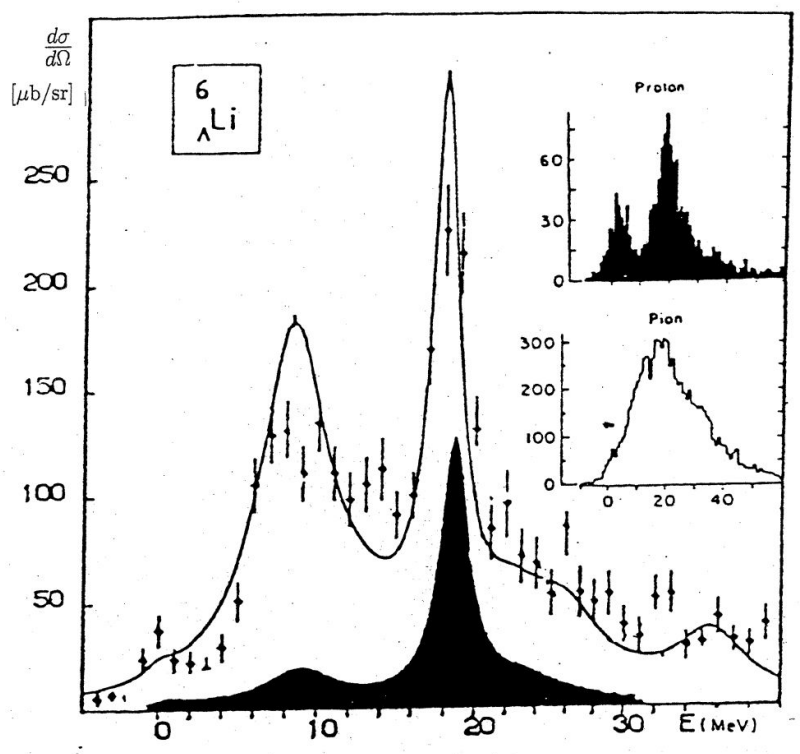

Figure 4: Spectrum of the ${ }_{\Lambda}^{6} \mathrm{Li}$ seen in $\left(K_{\text {in-flight }}^{-}, \pi^{-}\right)$

extended supermultiplet scheme which combines the $1 s$ and $1 p$ orbitals, and classifies the nuclear and hypernuclear states by Young tableaux $[f]$. The lower state has symmetry [41] for its nuclear core, so its break-up to ${ }_{\Lambda}^{5} \mathrm{He}+p$ or to ${ }^{5} \mathrm{Li}+\Lambda$ is allowed both energetically and by supermultiplet symmetry. The upper (narrow) state has symmetry $[f]=[32]$ for its nuclear core, so that its decay to ${ }^{5} \mathrm{Li}+\Lambda$ or ${ }_{\Lambda}^{5} \mathrm{He}+p$, is forbidden by the selection rules for the supermultiplet symmetry.

Another example of the important role $s^{-1} s_{\Lambda}$ states play are $\gamma$-ray transitions in hyperfragments. Gamma-rays from ${ }_{\Lambda}^{7} \mathrm{Li}$ formed by ${ }^{3} \mathrm{He}$ emission from highly excited states of ${ }_{\Lambda}^{10} \mathrm{~B}$ : $\mid s^{3} p^{6}[432] D ; s_{\Lambda}>$, were observed in the experiment ${ }^{10} \mathrm{~B}\left(K^{-}, \pi^{-} \gamma\right)$ [20]. Again, supermultiplet symmetry forbids the decay through the channels ${ }_{\Lambda}^{9} \mathrm{Be}+p$ and ${ }_{\Lambda}^{5} \mathrm{He}+\alpha+p$ because they correspond to $[f]=[44]$. 


\subsection{Excitation spectra}

We deciphered energy spectra for primary $p$-shell hypernuclei from reaction $\left(\pi^{+}, K^{+}\right)[\mathbb{L D}]$ as multiplets of $0 \hbar \omega$ and $1 \hbar \omega$ configurations e.g. $p^{-1} s_{\Lambda}, p^{-1} p_{\Lambda}$ and $s-1 s_{\Lambda}$. For example, every peak in spectrum of ${ }_{\Lambda}^{9} \mathrm{Be}$, Fig. [ ] is linked to one particular configuration ${ }^{8} \mathrm{Be}^{*} \otimes \ell_{\Lambda}$ in Table $\square$.

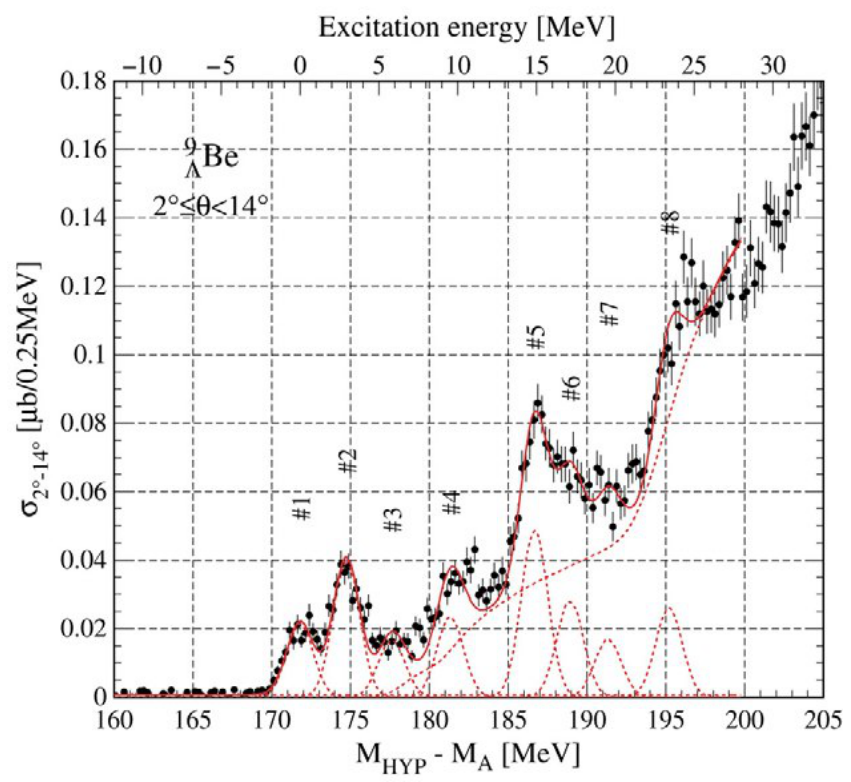

Figure 5: Excitation spectrum of ${ }_{\Lambda}^{9} \mathrm{Be}$ measured using the $\left(\pi^{+}, K^{+}\right)$reaction [20]

Table 2: Deciphering of the ${ }_{\Lambda}^{9} \mathrm{Be}$ spectrum

\begin{tabular}{|lr|crc|c|}
\hline \multicolumn{2}{|c|}{ experiment [20] } & \multicolumn{3}{|c|}{${ }^{9} \mathrm{Be}$} & \\
No & \multicolumn{1}{|c|}{$\mathrm{E}_{\mathrm{ex}}$} & $\mathrm{E}\left({ }^{8} \mathrm{Be}\right)$ & [40] & ${ }^{8} \mathrm{Be} \otimes l_{\Lambda}$ & $l_{n}^{-1} l_{\Lambda}$ \\
\hline 1. & 0.00 & 0.0 & & $s^{4} p^{4}[44] \mathrm{S} \otimes s_{\Lambda}$ & $p^{-1} s_{\Lambda}$ \\
2. & 2.93 & 3.0 & & $s^{4} p^{4}[44] \mathrm{D} \otimes s_{\Lambda}$ & $p^{-1} s_{\Lambda}$ \\
3. & 5.80 & & 0.0 & $s^{4} p^{4}[44] \otimes p_{\Lambda}:(50)$ & $p^{-1} p_{\Lambda}$ \\
4. & 9.52 & & 0.0 & $s^{4} p^{4}[44] \otimes p_{\Lambda}:(31)$ & $p^{-1} p_{\Lambda}$ \\
5. & 14.88 & 16.7 & & $s^{4} p^{4}[431] \mathrm{D} \otimes s_{\Lambda}$ & $p^{-1} s_{\Lambda}$ \\
6. & 17.13 & 18.9 & & $s^{3} p^{5}[44] \mathrm{P} \otimes s_{\Lambda}$ & $s^{-1} s_{\Lambda}$ \\
7. & 19.54 & & 16.7 & $s^{4} p^{4}[431] \mathrm{D} \otimes p_{\Lambda}$ & $p^{-1} p_{\Lambda}$ \\
8. & 23.40 & 24.0 & & $s^{3} p^{5}[431] \mathrm{P} \otimes s_{\Lambda}$ & $s^{-1} s_{\Lambda}$ \\
\hline
\end{tabular}




\subsection{Model}

Further, we use a notion of Strangeness Analogue State (SAS) introduced by Kerman and Lipkin [42]. They suggested that in $\left(K_{\text {in-flight }}^{-}, \pi^{-}\right)$reaction $\Lambda$ only replaces neutron in each shell model state. So, it combines a simple theoretical structure with a source of rich and easily analyzed data of resonances. This notion simplifies the interpretation of hypernuclear spectra, so, they are amenable to hand calculation.

For $1 p$-shell hypernuclei SAS do belong to the nominally " $1 \hbar \omega "$ excitation band splitted into three groups, because the single-particle energies differ significantly, as can be seen in Table B]. The situation reminds "configurational splitting" of Giant Dipole Resonance [43]].

Table 3: Three groups of $1 \hbar \omega$ excitation

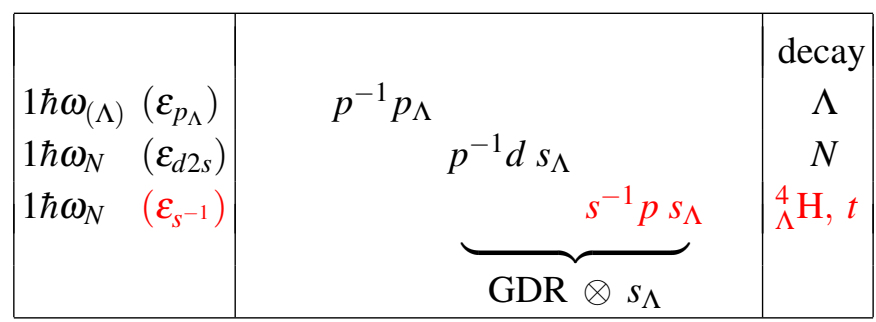

\subsection{TISM}

The wave functions are expanded on a basis set [4]

$$
\begin{gathered}
\Psi=\sum_{i} a_{i} \Phi_{i} . \\
\Phi_{i}=\left|l^{k_{1}}\left[f_{1}\right](\lambda \mu)_{1} l^{k_{2}}\left[f_{2}\right](\lambda \mu)_{2}:\left[f_{i}\right](\lambda \mu)_{i} L_{i} K_{i}>\cdot\right|[\tilde{f}] \beta S_{i} T_{i}>
\end{gathered}
$$

In this supermultiplet basis, $(\lambda \mu) L K$ label representations of $S U(3) \supset R_{3}$ in the orbital space, and $[\tilde{f}] \beta S T$ label representations of $S U(4) \supset S U(2) \times S U(2)$ in the spin-isospin space.

This basis turns out to be very good in the sense that wave functions for low-energy states $(0 \hbar \omega)$ are dominated by one basis state. Moreover, this basis permits the exclusion of spurious center-ofmass states in rather simple way. In standard shell model, among the $1 \hbar \omega$ basis configurations there are also "spurious states" of center-of-mass excitations $\Psi_{1}\left(R_{A}\right)$. In the Translation Invariant Shell Model (TISM) basis, we use the proper combination of "standard" shell model wave functions, in which w.f. of center of mass is either $\Psi_{0}\left(R_{A}\right)$ or $\Psi_{1}\left(R_{A}\right)$. The method is rather simple for wave functions written in LS basis. It is obvious, that the operator of center-of-mass, $\mathscr{R}$, does not change the supermultiplet structure $[f]$, but $(\lambda \mu)_{\text {spur }}=(10) \times(\lambda \mu)_{0}$.

First step is listing of all possible $[f],(\lambda \mu)_{0}$ for configurations $\mid s^{4} p^{k}>\equiv \Phi_{k}^{(A)}(k=A-4)$.

The second step is listing of possible configurations $\mid s^{4} p^{k-1}(2 s-d)>$ and $\mid s^{3} p^{k+1}>$ and spurious states $\mathscr{R} \Phi_{k}^{(A)}$ - the rest are pure configurations $\Phi_{k+1}^{(A)}$.

The last step, determination of the structure of spurious and pure states may be completed easily for configurations $\mid s^{3} p^{k}:[f]>$ : the $\alpha_{s p}$, weight of spurious component in configuration $\mid s^{3} p^{k}:[f]>$, is determined by fractional parentage decomposition of $\Psi_{0}\left(R_{A}\right) \cdot \Phi_{k}^{(A)}([f] \lambda \mu)$. 


$$
\begin{aligned}
& \Phi_{k}^{(A)} \Psi_{0}\left(R_{A}\right) \equiv\left|s^{4} p^{k}\left[f_{p}\right]:[f]=\left[4 f_{p}\right]\right\rangle \\
& \left|s^{4} p^{k}\left[f_{p}\right]:[f]=\left[4 f_{p}\right]\right\rangle= \\
& \sum_{f^{\prime}} \sqrt{\frac{n_{f}}{n_{f^{\prime}}}}\left\{\quad g_{p}\left|s^{4} p^{k-1}\left[f_{p}^{\prime}\right]:\left[f^{\prime}\right]\right\rangle|p\rangle \quad+g_{s}\left|s^{3} p^{k}:\left[f^{\prime}\right]\right\rangle|s\rangle \quad\right\} \\
& =\quad g_{p} \Phi_{k-1}^{(A-1)} \Psi_{0}\left(R_{k-1}\right) \psi_{1}(r) \\
& +\alpha_{s p} g_{s} \Phi_{k-1}^{(A-1)} \Psi_{1}\left(R_{k-1}\right) \psi_{0}(r) \quad+\beta g_{s} \Phi_{k}^{(A-1)} \Psi_{0}\left(R_{k-1}\right) \psi_{0}(r) \\
& \begin{array}{lll}
\Psi_{1}\left(R_{A}\right) \varphi_{0} & \Psi_{0}\left(R_{A}\right) \varphi_{1} & \Psi_{0}\left(R_{A}\right) \varphi_{0}
\end{array} \\
& g_{p} \sqrt{\frac{1}{A}} \quad-g_{p} \sqrt{\frac{A-1}{A}} \\
& +\alpha_{s p} g_{s} \sqrt{\frac{A-1}{A}} \quad \alpha_{s p} g_{s} \sqrt{\frac{1}{A}} \quad \beta g_{s} \\
& =0 \quad \Rightarrow \quad \alpha_{s p}=-\frac{g_{p}}{g_{s}} \sqrt{\frac{1}{A-1}}
\end{aligned}
$$

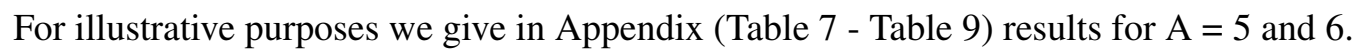

\subsection{TISM CFP}

The key ingredients in the shell model are coefficients of fractional parentage (CFP).

With one-particle CFP in hand we can write down components of SAS, (see Table $₫$ ).

Table 4: SAS

\begin{tabular}{|cccc|}
\hline target & $n_{f}$ & $g_{l}$ \\
\hline${ }^{6} \mathrm{Li}: \mid s^{4} p^{2}[42](20)>$ & $\frac{4}{9}$ & $\frac{9}{10}$ & $\Phi_{1}[41] \otimes p_{\Lambda}$ \\
& & $\frac{1}{10}$ & $\Phi_{2}[41] \otimes s_{\Lambda}$ \\
& $\frac{5}{9}$ & 1 & $\Phi_{2}[32] \otimes s_{\Lambda}$ \\
${ }^{7} \mathrm{Li}: \mid s^{4} p^{3}[43](30)>$ & $\frac{9}{14}$ & $\frac{7}{9}$ & $\Phi_{2}[42] \otimes p_{\Lambda}$ \\
& & $\frac{2}{9}$ & $\Phi_{3}[42] \otimes s_{\Lambda}$ \\
& $\frac{5}{14}$ & 1 & $\Phi_{3}[33] \otimes s_{\Lambda}$ \\
${ }^{9} \mathrm{Be}: \mid s^{4} p^{5}[441](31)>$ & $\frac{1}{6}$ & $\frac{15}{16}$ & $\Phi_{4}[44] \otimes p_{\Lambda}$ \\
& & $\frac{1}{16}$ & $\Phi_{5}[44] \otimes s_{\Lambda}$ \\
& $\frac{5}{6}$ & $\frac{9}{16}$ & $\Phi_{4}[431] \otimes p_{\Lambda}$ \\
& $\frac{7}{16}$ & $\Phi_{5}[431] \otimes s_{\Lambda}$ \\
\hline
\end{tabular}

To calculate Spectroscopic Amplitude (SA) for hyperfragment emission we need few-particle CFP. In TISM it reads:

$$
\begin{array}{r}
\Phi_{k}^{(A)}[f](\lambda \mu)=\sum_{f_{1}, f_{2}} \sum_{k_{1}, k_{2}, v} \sqrt{\frac{n f_{1} \cdot n f_{2}}{n f}} \Phi_{k_{1}}^{\left(A_{1}\right)}\left[f_{1}\right](\lambda \mu)_{1} \\
\Phi_{k_{2}}^{\left(A_{2}\right)}\left[f_{2}\right](\lambda \mu)_{2} \\
\varphi_{v}\left(R_{1}-R_{2}\right)
\end{array}
$$


Wave functions for "usual" clusters $(d, t, \alpha)$ are $\mid s^{k}>\equiv \Phi_{0}^{(k)}[k]$.

For heavy Hydrogen isotopes we take:

$$
\begin{array}{ll}
\mid s^{3} p:[31]>\equiv \Phi_{1}^{(4)}[31] & \left({ }^{4} \mathrm{H}\right), \\
\mid s^{3} p^{2}:[32]>\equiv \Phi_{2}^{(5)}[32] & \left({ }^{5} \mathrm{H}\right) .
\end{array}
$$

Spectroscopic amplitude for hyperfragment is multiplied by factor $\frac{A}{A-1} \cdot \frac{A_{c}}{A_{c}+1}$, reflecting Jacobi coordinates transformation.

\section{Predictions}

Now we present first results for lightest $p$-shell hypernuclei, ${ }_{\Lambda}^{6} \mathrm{He}$ and ${ }_{\Lambda}^{7} \mathrm{He}$. Spectroscopic amplitudes for emission $\Lambda$ and $n$ are zero for configurations $\mid s^{3} p^{k}[3 k] s_{\Lambda}>$.

Table 5: Decays of ${ }_{\Lambda}^{6} \mathrm{He}$

Decay modes \& thresholds

\begin{tabular}{|c|c|c|}
\hline${ }_{\Lambda}^{4} \mathrm{He}$ & ${ }_{\Lambda}^{5} \mathrm{He}$ & ${ }_{\Lambda}^{6} \mathrm{He}$ \\
$2 n$ & $n$ & $\Lambda$ \\
21.47 & 0.17 & 4.18 \\
\hline${ }_{\Lambda}^{3} \mathrm{H}$ & ${ }_{\Lambda}^{4} \mathrm{H}$ & ${ }_{\Lambda}^{5} \mathrm{H}$ \\
$t$ & $d$ & $p$ \\
20.85 & 18.94 & 22.86 \\
\hline
\end{tabular}

SA for $s_{\Lambda} \otimes \Phi_{2}^{(5)}[32]{ }^{24} S$

\begin{tabular}{|c|c|c|c|}
\hline channel & {$\left[f_{i}\right]\left[f_{k}\right]$} & $\mathrm{E}_{\text {th }}$ & $\mathrm{SA}$ \\
\hline${ }_{\Lambda}^{5} \mathrm{He}+n$ & {$[4][1]$} & 0.17 & 0 \\
${ }^{5} \mathrm{He}+\Lambda$ & {$[41]$} & 4.18 & 0 \\
${ }_{\Lambda}^{4} \mathrm{H}+d$ & {$[3][2]$} & 18.94 & 0.153 \\
${ }_{\Lambda}^{3} \mathrm{H}+t$ & {$[2][3]$} & 20.85 & 0.352 \\
\hline
\end{tabular}

Table 6: Decays of ${ }_{\Lambda}^{7} \mathrm{He}$

Decay modes and thresholds

\begin{tabular}{|c|c|c|c|}
\hline${ }_{\Lambda}^{4} \mathrm{He}$ & ${ }_{\Lambda}^{5} \mathrm{He}$ & ${ }_{\Lambda}^{6} \mathrm{He}$ & ${ }_{\Lambda}^{7} \mathrm{He}$ \\
$3 n$ & $2 n$ & $n$ & $\Lambda$ \\
21.1 & 3.1 & 2.2 & 5.2 \\
\hline${ }_{\Lambda}^{3} \mathrm{H}$ & ${ }_{\Lambda}^{4} \mathrm{H}$ & $\left({ }_{\Lambda}^{5} \mathrm{H}\right)$ & ${ }_{\Lambda}^{6} \mathrm{H}$ \\
$t n$ & $t$ & $d$ & $p$ \\
23.7 & 15.5 & & 23.8 \\
\hline
\end{tabular}

$\mathrm{SA}$ for $s_{\Lambda} \otimes \Phi_{3}^{(6)}[33]^{33} \mathrm{~S}$

\begin{tabular}{|c|c|c|c|}
\hline channel & {$\left[f_{i}\right]\left[f_{k}\right]$} & $\mathrm{E}_{\text {th }}$ & $\mathrm{SA}$ \\
\hline${ }_{\Lambda}^{6} \mathrm{He}+n$ & {$[41][1]$} & 2.82 & 0 \\
${ }_{\Lambda}^{5} \mathrm{He}+2 n$ & {$[4][1][1]$} & 3.08 & 0 \\
$\Lambda+{ }^{6} \mathrm{He}$ & {$[42]$} & 5.23 & 0 \\
${ }_{\Lambda}^{4} \mathrm{H}+t$ & {$[3][3]$} & 15.49 & 0.183 \\
${ }_{\Lambda}^{4} \mathrm{He}+3 n$ & {$[3][1][1][1]$} & 21.14 & 0 \\
${ }_{\Lambda}^{3} \mathrm{H}+t n$ & {$[2][3][1]$} & 23.66 & 0.214 \\
${ }_{\Lambda}^{6} \mathrm{H}+p$ & {$[32][1]$} & 23.81 & 0.101 \\
\hline
\end{tabular}




\section{Summary}

We present one approach to build model for identification hyperfragments based on Translation Invariant Shell Model, and Strange Analogue State. Model selection affects the complexity of calculation. Complexity of calculation may be resolved by numerical methods or by constraint satisfaction method. As a first step we use our knowledge to simplify the calculations and reduce "unimportant" states. So, we try to remain in algebraic solving knowledge based reduced problem. For light hypernuclei it is possible.

We explore simple "toy model" (notion of Strange Analog State [4]2]) to analyze cluster decay of primary hypernuclei. It is an extension of a very successful analysis of $\gamma$-transitions by Millener [B]]. At the beginning we focused attention on $s^{-1} s_{\Lambda}$ components of SAS only. The complete treatment is in progress. The results may be useful in judicious choice of targets as paper by Dalitz and Gal [34] was at dawn of hypernuclear $\gamma$ spectroscopy.

Such approach is complementary to transport model by Botvina et al. [44], [ए6].

Final remark. In J-Lab experiment [U] it is possible to identify unstable and even neutral hypernuclei [44]]. On the other hand, setup in Dubna [ㅍ], is well suited for identification of such exotic hypernucleus as ${ }_{\Lambda}^{8} \mathrm{H}$.

\section{Acknowledgement}

We would like to express our sincere thanks to P. Bydžovský and Liguang Tang inviting us to this challenging project, S.N. Nakamura and J. Pochodzalla for their interest in even preliminary result. Special thanks are due to John Millener for his expertise. 


\section{Appendix: TISM}

In Tables below results for $\mathrm{A}=5$ and 6 are given.

Table 7: Configuration list for $\mathrm{A}=5$

\begin{tabular}{|rl|l|l|l|}
\hline & {$[f]:$} & \multicolumn{1}{|c|}{$[41]$} & {$[32]$} & {$[311]$} \\
\hline $\mathrm{N}=1$ & $s^{4} p \equiv \Phi_{1}$ & $(10)$ & & \\
\hline $\mathrm{N}=2$ & $s^{4} d$ & $(20)$ & & \\
& $s^{3} p^{2}[2]$ & $(20)$ & $(20)$ & \\
& $s^{3} p^{2}[11]$ & $(01)$ & & $(01)$ \\
\cline { 2 - 4 } & $\mathscr{R} \Phi_{1}$ & $(20)(01)$ & & \\
$\Phi_{2}$ & $(20)$ & $(20)$ & $(01)$ \\
\hline
\end{tabular}

TISM structure

\begin{tabular}{|c|c|c|c|c|}
\hline $\begin{array}{l}{[f]} \\
(\lambda \mu)\end{array}$ & $\begin{array}{c}{[41]} \\
(20) \\
\mathscr{R} \Phi_{1} \Phi_{2}\end{array}$ & $\begin{array}{l}{[41]} \\
(01) \\
\mathscr{R} \Phi_{1}\end{array}$ & $\begin{array}{l}{[32]} \\
(20) \\
\Phi_{2}\end{array}$ & $\begin{array}{c}{[311]} \\
(01) \\
\Phi_{2}\end{array}$ \\
\hline $\begin{array}{l}s^{4} d \\
s^{3} p^{2}\end{array}$ & $\begin{array}{r}\sqrt{\frac{2}{5}} \sqrt{\frac{3}{5}} \\
-\sqrt{\frac{3}{5}} \sqrt{\frac{2}{5}}\end{array}$ & 1 & 1 & 1 \\
\hline
\end{tabular}

Table 8: Configuration list for $\mathrm{A}=6 \quad \mathrm{~N} \leq 3$

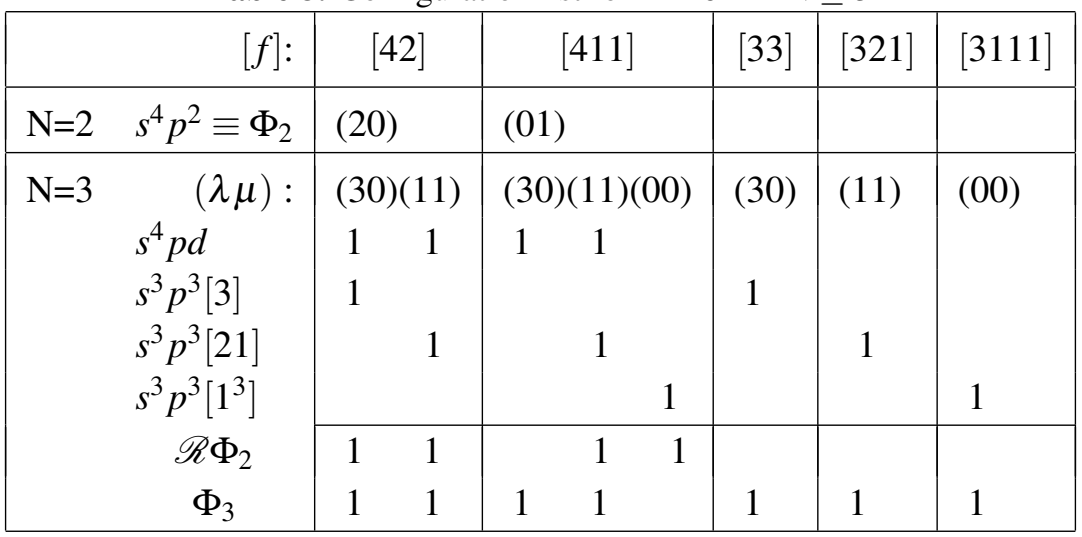

Table 9: TISM structure for $A=6, N=3$

\begin{tabular}{|c|c|c|c|c|c|c|c|c|}
\hline $\begin{array}{r}{[f]:} \\
(\lambda \mu):\end{array}$ & $\begin{array}{c}{[42]} \\
(30) \\
\mathscr{R} \Phi_{2} \Phi_{3}\end{array}$ & $\begin{array}{c}{[42]} \\
(11) \\
\mathscr{R} \Phi_{2} \Phi_{3}\end{array}$ & $\begin{array}{c}{[411]} \\
(30) \\
\Phi_{3} \\
\end{array}$ & $\begin{array}{c}{[411]} \\
(11) \\
\mathscr{R} \Phi_{2} \quad \Phi_{3}\end{array}$ & $\begin{array}{l}{[411]} \\
(00) \\
\mathscr{R} \Phi_{3} \\
\end{array}$ & $\begin{array}{c}{[33]} \\
(30) \\
\Phi_{2} \\
\end{array}$ & $\begin{array}{c}{[321]} \\
(11) \\
\Phi_{3} \\
\end{array}$ & $\begin{array}{c}{[3111]} \\
(00) \\
\Phi_{3} \\
\end{array}$ \\
\hline $\begin{array}{l}s^{4} p d \\
s^{3} p^{3}[3] \\
s^{3} p^{3}[21] \\
s^{3} p^{3}\left[1^{3}\right]\end{array}$ & $\begin{array}{r}\sqrt{\frac{4}{6}} \sqrt{\frac{2}{6}} \\
-\sqrt{\frac{2}{6}} \sqrt{\frac{4}{6}}\end{array}$ & $\begin{array}{r}\sqrt{\frac{1}{6}} \sqrt{\frac{5}{6}} \\
-\sqrt{\frac{5}{6}} \sqrt{\frac{1}{6}}\end{array}$ & 1 & $\begin{array}{c}\sqrt{\frac{3}{6}} \sqrt{\frac{3}{6}} \\
\sqrt{-\frac{3}{6}} \sqrt{\frac{3}{6}}\end{array}$ & 1 & 1 & 1 & 1 \\
\hline
\end{tabular}




\section{References}

[1] L. Tang, et al.: Study of light hypernuclei by pionic decay.

Proposal E-10-001, Jefferson Lab.

[2] P. Auerbach et al. (A1 Collaboration), Nucl. Phys. A881, 187 (2012).

[3] H. Tamura et al., Nucl. Phys. A881, 310 (2012).

[4] M. Danysz and J. Pniewski, Phil. Mag. 44, 348 (1953).

[5] K.J. Nield et al., Phys. Rev. C13, 1263 (1976).

[6] A. Abdurakhimov et al., N. Cim. 102A, 645 (1989);

S. Avramenko et al., Nucl. Phys. A547, 95c (1992).

[7] B.I. Abelev et al. (STAR Collaboration), Science 328, 58 (2010).

[8] A.G. Reuber, K. Holinde, J. Speth, Czech. J. Phys. 42, 1115 (1992).

[9] Th.A. Rijken, Nucl. Phys. A691, 322c (2001).

[10] D.J. Millener et al., Phys. Rev. C31, 449 (1985).

[11] V.N. Fetisov et al., Z. Phys. A339, 399 (1991).

[12] S.R. Beane et al., arXiv:1206.5219

[13] E. Botta, T. Bressani, G. Garbarino, E. Phys. J. A48, 41 (2012).

[14] T.R. Saito et al., Nucl. Phys. A881, 218 (2012).

[15] A.V. Aver'yanov et al., Phys. At. Nucl. 71, 2101 (2008).

[16] J. Pochodzalla, Acta Phys. Polon. B 42, (2011) 833.

[17] R.E. Chrien, C.B. Dover, Ann. Rev. Nucl. Part. Sci. 39, 113 (1989).

[18] T. Hagesawa et al., Phys. Rev. C53, 1210 (1996).

[19] F. Cusanno et al., Nucl. Phys. A835, 129 (2010).

[20] O. Hashimoto, H. Tamura, Progr. Part. Nucl. Phys. 57, 564 (2006).

[21] H. Tamura et al., Phys. Rev. Lett. 84, 5963 (2000).

[22] B. Jonson, Phys. Reports 389, 1 (2004).

[23] T. Bressani, in Proc. Workshop on physics and Detectors for DAФNE, Frascati, 1991.

[24] L. Majling, Nucl. Phys. A585, 211c (1995).

[25] T. Bressani, Proc. Int. School "E. Fermi", Course 153, p. 323 (2003).

[26] R.H. Dalitz, R. Levi Seti, N. Cimmento 30, 489 (1963).

[27] W. Gajewski et al., Nucl. Phys. B2, 288 (1967).

[28] M. Agnello et al., Phys. Lett. B640, 145 (2006).

[29] M. Agnello et al. (FINUDA Collaboration and A. Gal), Phys. Rev. Lett. 108, 042501 (2012);

Nucl. Phys. A881, 269 (2012).

[30] J. Lukstins, Nucl. Phys. A691, 491c (2001).

[31] L. Majling et al., Proceedings XVIII Int. Baldin Seminar, vol.2, 153 (2008).

[32] R.H. Dalitz, A. Gal, Nucl. Phys. B1, 1 (1967).

[33] A. Korsheninnikov et al., Phys. Rev. Lett. 90, 082501 (2003).

[34] R.H. Dalitz, A. Gal, Ann. Phys. 116, 167 (1978).

[35] D.J. Millener, Lecture Notes in Physics, 724, 31 (2007).

[36] E.H. Auerbach et al., Ann. Phys. 148, 381 (1983).

[37] J. Žofka et al.,: Phys.Part.Nucl. 22 (1991); 28 (1997) 101.

[38] R. Bertini et al., Nucl. Phys. A368, 365 (1981).

[39] L. Majling et al., Phys. Lett. 92B, 256 (1980).

[40] D.R. Tilley et al., Nucl. Phys. A708, 3 (2002).

[41] V. Kukulin, Yu. Smirnov, L. Majling, Nucl. Phys. A103, 681 (1967).

[42] A.K. Kerman, H.J. Lipkin, Ann. Phys. 66, 738 (1971).

[43] R. Eramzhyan et al., Physics Reports, 136, 229 (1986).

[44] A.S. Botvina et al., Nucl. Phys. A881, 228 (2012). 